\title{
A Recent Research on Non Alcoholic Fatty Liver Disease
}

\author{
Theivendren Panneerselvam, Deepak Chemmanthitta Gopinath, Bipin Shaji, Sankarganesh Arunachalam, \\ Selvaraj Kunjiappan
}

\begin{abstract}
Non alcoholic fatty liver disease (NAFLD) is a persistent condition of liver steatosis in the absence of alcohol consumption. The main risk factors associated with the disease are hypertension, diabetes mellitus and dyslipidemia these are together termed as metabolic syndrome. Recent studies identified that children are more vulnerable to develop NAFLD in future due to sedentary life styles and poor diet. NAFLD can further make complications of cardiovascular and liver related disorders. Apart from these causes there are factors such as enzyme, gene expression; amino acid regulation and so on in the human body plays an important role in the development of NAFLD. The objective of this research is to summaries the various ways by which a NAFLD is affected in a normal individual and to point out current research on treatment strategy for the NAFLD.
\end{abstract}

Keywords: NAFLD, Metabolic syndrome, Management of NAFLD.

\section{INTRODUCTION}

NAFLD is the presence of fatty liver disease without the consumption of alcohol or any other hepatotoxic medications and the main causes of NAFLD are diabetes mellitus, dyslipidemia and obesity. ${ }^{1}$ A NAFLD further leads to liver damage and results in liver cancer. A NAFLD is classified into the non-alcoholic fatty liver (NAFL) and non-alcoholic steatohepatitis (NASH). In NAFL there is steatosis without hepatic injury on the other hand in NASH there is steatosis with a hepatic injury with or without fibrosis. It can also occur in lean persons with normal glucose and normal lipid profile. Diagnosis of NAFLD should be based on 4 criteria, i) There is no alcohol consumption, ii) Presence of hepatic steatosis by histology, iii) There is no competing aetiology for steatosis, iv) No evidence of coexisting chronic liver parenchymal disease like an autoimmune liver disease, viral hepatitis, hepatolenticular degeneration. NAFLD fibrosis score can be used to evaluate the patients diagnosed with NAFLD who are at an increased likelihood of getting affected with fibrosis and cirrhosis. NAFLD fibrosis score is based on BMI, triglyceride level, platelet count, albumin, aspartate transaminase (AST) and alaninine transaminase (ALT) ratio. Gold standard for the diagnosis of NAFLD is liver biopsy should be done in individuals with advanced steatosis because it is expensive. Many drugs are pharmacologically recommended but most of them are not efficacious in prolonged use.

Revised Manuscript Received on December 05, 2019

T. Panneerselvam Principal, Saraswathi College of Pharmacy,

Deepak C G, Pharm D Student, Karavali College of Pharmacy, Thiruvail, Karnataka

Bipin Shaji, Pharm D Student, Karavali College of Pharmacy, Thiruvail, Karnataka

A.Sankar Ganesh, Assistant Professor, Department of Biotechnology, Kalasalingam Academy of Research and Education, Krishnankoil, Tamil Nadu

K. Selvaraj, Assistant Professor, Department of Biotechnology, Kalasalingam Academy of Research and Education, Tamilnadu.
Orlistat in conjunction with lifestyle modification improved fatty liver and liver enzyme ALT levels although their contact on liver tissue could not be identified. Reducing body weight of 3-5\% helps to improve steatosis. Exercise can also help to improve steatosis in adults. Insulin-sensitizing agents like pioglitazone can be used in the therapy of biopsy-proven NAFLD. Vitamin E (alpha-tocopherol) 800IU/day is efficacious in improving steatosis and swelling but ineffective in fibrosis. Omega-3- fatty acids can be considered for the management of hypertriglyceridemia in NAFLD. ${ }^{2}$ In obese individuals, the rate of incidence of a NAFLD is $60 \%-90 \%$ while the occurrence of NASH and hepatic cirrhosis is $20 \%-25 \%$ and $2 \%-8 \%$ respectively. ${ }^{3}$ The main risk factors of the disease are (i) Obesity - central fat accumulation and (>102 cm in males and $>88 \mathrm{~cm}$ in females) and overweight (>29 kg/meter square) are predisposing factors for NAFLD, (ii) Altered glucose regulation, (iii) Hypertriglyceridemia $>2 \mathrm{mmol} / \mathrm{l}$, (iv) Hypertension $>160 / 95 \mathrm{mmHg}$, (v) Family history of diabetes - first degree relatives, (vi) Parentage of hypertension - first degree relatives, (vii) Cardiovascular disease, (viii) Parentage of cardiovascular disease - first degree relatives, (ix) Chronic infections- hepatitis $\mathrm{C}$ viral infections and human immunodeficiency virus. In children, cirrhosis is rarely reported. ${ }^{4}$ Recent studies identified that hyperinsulinemia and insulin resistance also responsible for the advancement of the NAFLD. ${ }^{1,5}$ The disease itself does not have any peculiar signs and symptoms but it is imperative to contemplate obese children. The characteristic features of the disease may vary in adults and children. The NAFLD does not have any specific treatment. ${ }^{6}$ The main reason for choosing this topic to review since the NAFLD is the most typical source of chronic parenchymal liver disease in a pediatric population within advanced countries. Data regarding the treatment of the disease is very less and new interventional discoveries are going in the present scenario about this topic. As the disease is found in the developed countries one can expect the disease in the developing nations like India in the upcoming years.

A.Study reports related to genetics and enzyme regulation in NAFLD

A study was performed to demonstrate that deposition of fat in the hepatocytes as occurring in the NAFLD causes a reversible decline in the function of urea cycle enzymes. These reductions in the enzymes lead to buildup of toxic substance ammonia. Growth of ammonia results in damage to the liver. The study is conducted in both rats and humans. Liver biopsies are obtained from the humans with NASH and hepatocytes are secluded from rats which are fed with NASH inducing diet.

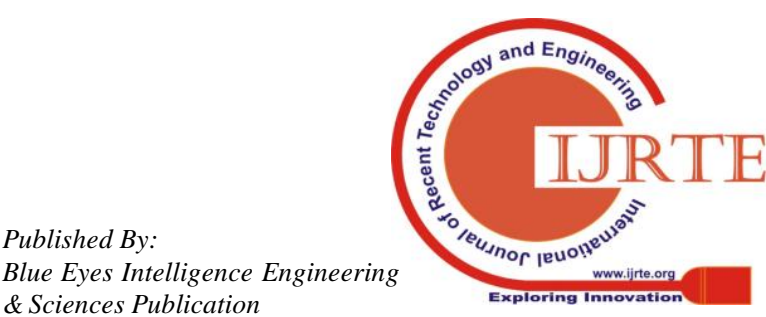




\section{A Recent Research on Non Alcoholic Fatty Liver Disease}

They estimated the concentrations of ammonia, ornithine transcarbamylase activity and gene expression in both the cases. From the study, De Chiara et al 2018. ${ }^{7}$ concluded that NAFLD is linked with fall in protein and gene expression and decline in the function of urea cycle enzymes resulting in increased accumulation of ammonia.

Bae CR et al 2018. ${ }^{8}$ studied the role of Endothelialcell-specific over expression of Endothelial C- type natriuretic peptide (E-CNP) in the livers affected with NASH. The study was performed in NASH induced mice by giving a high-fat diet (HFD) for 25 weeks or cholinedeficiency defined L- amino acid diet (CDAA) for 10 weeks. Then the mice were evaluated for (1) liver and serum parameters (2) blood sugar (3) hepatic fibrosis and inflammation (4) liver histology and (5) gene expression analysis. Data obtained was analyzed through Statistical Package for Social Sciences (SPSS) software version 12.0. At the end of the study they found that CNP in endothelial cells meliorates insulin tolerance, glucose tolerance, lipid profile and hepatic fibrosis in HFD induced NASH mice but in CDAA causing NASH, CNP in endothelial cell enhances glucose tolerance and ineffective in preventing hepatic fibrosis and inflammation.

$\mathrm{Xu} \mathrm{Y}$ et al 2018. ${ }^{9}$ investigated the linkage between the polymorphism of apolipoprotein A5 (APOA5) and the associated threat of NAFLD. The research was conducted in Chinese Han population. The study includes 232 NAFLD patients of possible single nucleotide polymorphisms (SNP) $\mathrm{s}$ of $A P O A 5$ and 188 healthy subjects. Biochemical methods were used for assessing the clinical characteristics. SPSS statistical software, version 20.0 was used. At the end, they found that the 5 genotypes of APOA5 were not associated with NAFLD. The genotypes showed response toward NAFLD. From this, they concluded that the polymorphism and NAFLD may be interlinked to each other.

The role of Nicotinamide adenine dinucleotide phosphate (NADPH) oxidase 1 (NOX1) isoform of NADPH oxidase in the disturbance in the function of liver sinusoids of patients with NAFLD was investigated by Matsumoto M et al 2018. ${ }^{10}$ It was identified that this NOX1 can cause advancement of liver fibrosis through the bile duct ligation. The research was conducted in both animals and as well as humans. Liver tissues were obtained from the NASH affected patients and healthy controls. Messanger riboxynucleic acid (mRNA) was isolated from these liver tissues and by using a kit from Bio-Rad (Hercules, CA) reverse transcription was demonstrated. For animal experiments, mice were used which fed HFD and high cholesterol diet for 8 weeks. Results were explicit as a mean \pm standard deviation. Statistical analyses used in the study include Student $t$-test, Analysis of Variance (ANOVA), and post-hoc Tukey-Kramer's test. In the end of the study, they found NOX1 mRNA levels were increased in NASH affected patients than healthy controls (human study) and in the animal model also the level of NOX1 was greatly elevated in HFD induced mice.

Yamada T et al 2018. ${ }^{11}$ show that by regenerating Parkin independent mitophagy pathway 1 can palliate the progression of NAFLD. Mitochondrial stasis helps to save hepatic injury and hypertrophy in a single knockout (KO). The investigators induced mitochondrial stasis by obstructing dynamin- related mitochondrial partition and combination by means of liver-specific KO mice for Drp1 and Opa1. They demonstrated that mitochondrial stasis re-launched mitochondrial mass and regenerates the normal rank of hepatic tissues. They also found that the autophagy adaptor protein p62/sequestosome-1 accelerates mitochondrial ubiquitination by enrolling Rbx1 and Keap1, two elements of acullin-RING E3 ligase complex, to mitochondria. An ideal mitochondrial size, which is not dependent on of division and fusion, regulates organ status and parkin-independent mitophagy in the liver.

Borai IH et al 2018. ${ }^{12}$ conducted a research to find out the association between plasma cell glycoprotein 1 (PC-1) K121Q gene polymorphism in the advancement of NAFLD. 87 NAFLD patients were enrolled in the experiment and categorized into 3 groups based on the grades in ultrasonography (USG). (1) Mild NAFLD, (2) moderate NAFLD, (3) severe NAFLD. Restriction fragment length polymorphism (RFLP) - Polymerized chain reaction (PCR) was used for the detection of PC-1 K121Q gene polymorphism. SPSS software was used for statistical analysis. In the results, they found that the lipid profile parameter is closely linked to the occurrence of NAFLD. The PC-1 level was greatly linked to triacylglycerol levels in NAFLD patients. From the study they established that the lipid profile parameters are predictive markers for the occurrence of NAFLD and triacylglycerol (TAG) level is the specific parameter in the pathology and likelihood of PC-1 gene polymorphism.

C1q/TNF- allied protein-3 (CTRP3) is a new adipokine that acts a vital role in regulating insulin sensitivity. A study was conducted by Zhou W et al 2018. ${ }^{13}$ to find out the part of CPRP3 in the progression of NAFLD. The research was conducted in a hospital and the adipokine levels are estimated using an ELISA. The prospective study was used in the current research. A total of 814 subjects at standard, 313 subjects were enrolled in follow-up. At standard CTRP3 levels was in patients with NAFLD than in non-NAFLD subjects. After a 3-year follow-up, the CTRP3 levels were decreased from the standard to the follow-up in the subjects who developed NAFLD. From the study, researchers concluded that there was an inverse relationship between CTRP3 and progression of NAFLD.

Protein tyrosine phosphatase 1B (PTB1B) is a negative regulator of insulin and cytokine signaling has a therapeutic role in T2DM. PTB1B mainly acts through insulin receptors (IR) and leptin receptors linked with Janus kinase 2 (JK2). Gonzalez- Rodriguez et al 2018. ${ }^{14}$ performed a research to assess the brunt of PTB1B deficiency in NASH. The experiments were performed in mice which divided into 2 groups (1) PTBIB wild-type (PTP1BWT) and (2) PTP1B deficient- (PTP1BKO) mice. Both the groups of mice had fed with methionine /choline-deficient diet (MCD) for 8 weeks. A control replica was introduced by substituting MCD with a normal diet for 1 week. After that, hepatic tissues of both the groups were analyzed. Researchers concluded that PTP1BWT mice showed NASH during the 8 weeks and elevation in the parameters such as Fibrosis Growth Factor (FGF)-21, Interleukin (IL)-6 and mRNAs. Parameters were declined when the diet was shifted to normal chow. But in PTP1BKO mice, there was an accelerated development of NASH

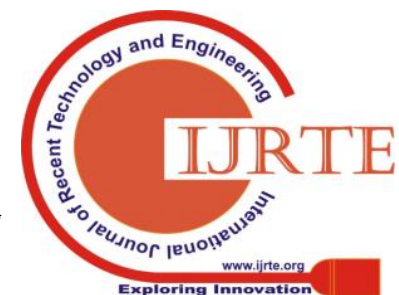


when the mice fed with MCD and return to normal in CHD. The investigators concluded that the PTB1B had a dual role i.e. development and reversion in NASH.

As per the hypothesis done by Seko Y et al $2018 .{ }^{15}$ they found that several genes are concerned with the progression of the disease. They suggested that the Patatin-like phospholipase domain containing protein 3 (PNPLA3) genes plays a role in stetosis, fibrosis and its progression to hepatocellular carcinoma. Along with other physiologic factors, genetic factors also associated with development and progression of NAFLD. So, it must be considered while diagnosing and managing NAFLD.

\section{B.Study reports related to biomarkers of NAFLD}

Visceral adiposity index (VAI) can also be used as a marker for NAFLD in premenopausal women with and without polycystic ovary syndrome. In the study, VAI is compared with 3 diagnostics indices- (1) Fatty Liver Index (FLI), (2) lipid accumulation product (LAP), and hepatic steatosis index (HSI).145 premenopausal women with the polycystic ovarian syndrome and 145 healthy control women with same senesce and BMI were included in the study. The methodology used was a cross-sectional retrospective study. Receiver Operating Characteristic (ROC) was used to measure the diagnostic performances of the 4 indices. From the study, they identified that VAI, FLI, LAP, HSI values are significantly high in women with NAFLD than those without NAFLD. They concluded that VAI was a useful marker in predicting NAFLD. The study was conducted by Vassilatou E et al 2018. ${ }^{16}$

The disparity between platelet count in NAFLD and liver cirrhosis with hepatitis $\mathrm{C}$ were examined by Ikarashi Y et al, 2018. ${ }^{17}$ They enrolled 620 NAFLD patients and 405 chronic liver diseases with hepatitis C patients in their study. Through their study they correlated the association between grade of fibrosis and platelet count in the mentioned disease. Finally, they observed that NAFLD patients are having elevated platelet count at each fibrosis grade than chronic liver disease with hepatitis $\mathrm{C}$ patients. They concluded as, the etiological aspects related to platelet reduction is unclear and it needs another analysis.

A study had been carried out to analyze the group of bio-markers which can be able to differentiate NAFLD from $\mathrm{NASH}$ and to inspect the molecular level of functioning associated with the activity of advancement of NASH from NAFLD. For their (Dong S et al , 2017. ${ }^{18}$ ) study the urine and blood samples were collected from 33 non diabetic subjects with NAFLD and steatosis, having normal liver function and 45 subjects who were having diagnosed with NASH having abnormal liver function. They also introduced 30 healthy subjects, whose urine and blood samples were collected and marked as control group in their study. The samples were evaluated to find out the possible non-invasive bio-markers. In their study they found out that there were abnormal liver function and elevated serum lipid concentration in NASH patients when correlated with NAFLD patients. The urinary metabolomics were varied by 31 metabolites. The variation in nucleic acids and amino acids levels are also seen. Finally, they concluded as, the bio-markers will help to distinguish NAFLD from NASH and also it helps to understand the molecular functions involved. The urinary bio-markers help in diagnosis, and to assess the effectiveness of treatment procedure. A study had been carried out by Hagstrom $\mathrm{H}$ et al, $2016{ }^{19}$ to identify whether serum high ferritin levels increases the death rate in NAFLD. They enrolled 222 individuals for their study. They had performed a cohort study and found out that high ferritin levels are associated with increased mortality rate.

\section{C.Study reports related to the prevalence and incidence of NAFLD}

A study was carried out by Dai W, et al, $2017 .^{20}$ to inspect the merged occurrence of NAFLD in Type 2 Diabetes Mellitus (T2DM) patients. In their Meta analysis, they included 35,599 T2DM patients associated from 24 studies. Out of this 20,264 were diagnosed with NAFLD. They found that the prevalence was about $29.6 \%$ to $87.1 \%$ and the merged occurrence of NAFLD in T2DM about $59.67 \%$. This study also suggested that incidence of NAFLD in T2DM were also varied accordingly by gender, obesity, dyslipidemia, coronary heart disease and chronic kidney disease, Finally, they concluded as there is a great correlation between the prevalence of NAFLD in T2DM patients, so it is important to evaluate the T2DM patients and screening of NAFLD done accordingly for its proper management and for targeted effects.

A research had done by Bellentani.S et al, $2010 .{ }^{21}$ to analyze the incidence of NAFLD. In Western countries the prevalence of NAFLD in common population is about $20-30 \%$. The prevalence is higher in male and increases with age. About 2-3\% of NAFLD patients are having NASH and it may progress to liver cirrhosis and hepatocellular carcinoma. According to the Dallas Heart study and the Dionysos study, $30 \%$ of the in USA and $25 \%$ in Italy have NAFLD. In these studies, about $79 \%$ and $55 \%$ of patients with NAFLD had normal amino transferase level, indicates that liver enzymes are not surrogate markers of NAFLD. In children the prevalence of NAFLD is about $3-10 \%$ which rises to about 40-70\% among obese children Fig 1.

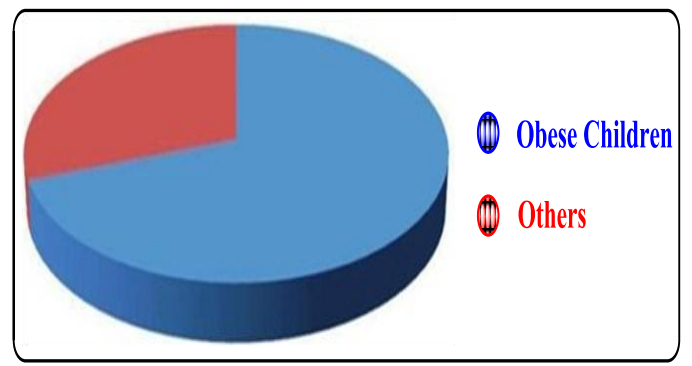

Fig 1: Prevalence of NAFLD in children. The prevalence of NAFLD in children is about $3-10 \%$ and its incidence increases about $\mathbf{4 0 - 7 0 \%}$ in obese children.

\section{D.Study reports related to the risk factors and etiology of NAFLD}

Van den Berg EH et al 2018. ${ }^{22}$ performed a study to identify the association among cholesterol efflux capacity with NAFLD. They conducted a study with 639 subjects among that 36 subjects were affected with T2DM and 226 subjects 


\section{A Recent Research on Non Alcoholic Fatty Liver Disease}

with metabolic syndrome. FLI greater than or equal to 60 is used as the substitute for NAFLD. At the closing stages of the study, more than half of the participants had FLI greater than or equal to 60 .

From the study, they concluded that cholesterol efflux capacity is linked with NAFLD and reduced cholesterol efflux pumps are one of the etiology for myocardial disease in NAFLD patients.

Li TT et al $2018 .^{23}$ conducted a study in recently diagnosed diabetes in Chinese patients with contrast the distinctiveness of NAFLD in type 1 diabetes (T1DM), ketosis-onset and non-ketotic T2DM patients. The method of study used in the research was a cross-sectional study. The study includes 39 patients with T1DM, 27 patients with ketosis onset, 173 patients with non-ketotic T2DM, and 30 patients with non-diabetic individuals. The results of the study show no differences in the prevalence and risk factors between ketosis-onset diabetes and non-ketotic T2DM. In both these cases, BMI and ALT were found to be the sovereign etiologies. But in T1DM uric acid was identified as a self-reliant cause. urine $\mathrm{pH}$ and the occurrence of NAFLD. The study consists of 11,012 subjects including both the genders. Exclusion criteria involved men consumed ethanol more than $60 \mathrm{~g} /$ day and women more than $40 \mathrm{~g} /$ day. The participants are then classified into 3 groups based on the urine $\mathrm{pH}$. (1) Urine $\mathrm{pH}$ less than or equal to 5.5, (2) urine $\mathrm{pH}$ equals 6.0 , (3) urine $\mathrm{pH}$ greater than or equal to 6.5. After the prescribed span of time, 2023 volunteers developed NAFLD. From the study, Okamura $\mathrm{T}$ et al 2018. ${ }^{24}$ concluded that lowest urine $\mathrm{pH}$ shows higher liability occurrence of NAFLD than higher urine $\mathrm{pH}$.

The relationship between glucose regulation and ultrasound detected NAFLD in T2DM was studied by Afolabi BI et al $2018 .^{25} 80$ subjects aged 40-80 years with T2DM were involved. Subject's livers were assessed by B- mode ultrasound. Statistical analysis used in the study was SPSS version $20.68 \%$ of patients (55 patients) were recognized with NAFLD USG grades I (16.3\%), II (32.5\%) and III (20.0\%). $37.2 \%$ of subjects had grade 0 . From the study, the investigators concluded that obesity was the strong prognosticator of NAFLD and poor glucose regulation was the moderate seer of NAFLD.

Dowla S et al $2018 .^{26}$ studied on NAFLD diagnosed children to correlate the impact of carbohydrate-restricted diet vs. fatrestricted diet, ongoing accepted care, changes in the fatty infiltration of the liver, body composition and health over 8 weeks. Method of the study was prospective, two-arm, parallel, randomized controlled trial. Children between the ages of 9-18 years were considered in the research. Magnetic resonance spectroscopy and other blood tests were used to measure the hepatic lipid content, liver transaminases, visceral fat, dietary intake and adherence and markers of inflammation. From the study, they identified that it is critical to limit the total amount of energy produced by carbohydrate sources to below $40 \%$ to improve steatosis and other metabolic abnormalities in children in addition to carbohydrate diet restriction.

Wang L et al 2018. ${ }^{27}$ investigated the association between cardiovascular health and NAFLD. It was a prospective
A prospective study was done to find out the guild between

cohort study. The study included 3,424 subjects aged $>40$ years without NAFLD and they were followed up to 5 years. Liver USG was used to diagnose NAFLD. Smoking, physical activity, BMI, lipid profile, BP, and fasting glucose were used to estimate cardiovascular health condition. After 5 years they noted that optimum and conservative cardiovascular health was the guild to $37 \%$ alleviation in NAFLD and low fibrosis score compare to poor cardiovascular health. From the study, they established that ideal cardiovascular disease health was inversely associated with NAFLD.

Chou YT et al 2018. ${ }^{28}$ performed a study in Taiwanese population to screen the guild of sleep length and value with NAFLD by sexual characteristics. 6663 subjects aged greater than 18 years were volunteered for the study. With the help of USG, the harshness of NAFLD was alienated into mild, moderate and severe. Sleep duration was categorized into 3 based on hours of sleep i.e. short $(<6 \mathrm{~h})$, normal $(6-8 \mathrm{~h})$, and long $(>8 \mathrm{~h}$ ). The value of the sleep was assessed by using the Pittsburgh Sleep Quality Index (PSQI). In the end, they found that sleep state was not connected to NAFLD in females but it was negatively linked with males with mild and moderate-severe NAFLD. They established that a male with the poor quality of sleep was associated with alleviated risk of NAFLD.

Chang HT et al $2018 .^{29}$ performed a research to evaluate the expected causes and conceivable defensive factors for tamoxifen- associated NAFLD in breast cancer patients. The study was a retrospective cohort study. All patients involved in the study were aged between 20-70 years. Patients with alcoholic hepatitis, liver metastasis, chemotherapy-induced liver disease, and those patients who received non-tamoxifen hormonal therapy and patients who used tamoxifen less than 3 months were excluded. They also evaluated BMI, body height, weight, waist circumference. The researcher also documented the patient's manner of living after the diagnosis of a breast tumor. Statistical analysis used was found to be Statistics and Data (STATA) software v.13.0. From the study, they identified that the threat of the tamoxifenassociated fatty liver is more significant in patients with BMI over $22 \mathrm{~kg} / \mathrm{m}^{2}$ and a past history of hyperglycemia.

Patients with NAFLD who are involved in binge alcohol use showed less improvement in steatosis and steatohepatitis. Patients diagnosed with NAFLD aged above 21 years were integrated in the study. From those patients records of their liver biopsies and alcohol use in the time gap of 2 years were collected. In every follow-up, the subjects were enquired about (1) frequency of alcohol consumption, (2) quantity of drinks per day, and (3) rate of heavy drinking. Logistic regression analysis was used for assessing the guild among alteration in drinking type and modification in histology and ANOVA was used for evaluating the relationship among baseline drinking type and alteration in fibrosis stage, NASH tissue examination, and the NAFLD Activity Score and its entities. From the study, they identified that non-drinkers had the greatest reduction in steatosis grade and AST than binge drinkers. It explains that binge alcohol consumption was connected with fewer enhancements in steatosis and stage of AST. This study was performed by Ajmera V et al $2018 .^{30}$ 
Liu $\mathrm{P}$ et al 2018. ${ }^{31}$ performed a research to probe the modification status of NAFLD with the threat of hypertension and vice versa. Dongfeng-Tongji was the methodology used in the study. About 6704 hypertension free subjects and 9328 NAFLD free subjects were enrolled in the study. The statistical analysis used in the study was Statistical Analysis System (SAS) software.

At the end of the study, out of 6704 subjects free of hypertension 2561 subjects developed hypertension and 2289 developed NAFLD out of 9328 NAFLD free subjects. From the study, the investigators concluded that occurrence and the perseverance of NAFLD are linked with increased risk of hypertension.

Pattnaik K et al 2018. ${ }^{32}$ conducted a study in eastern coastal India to discover the aetiology linked with liver fibrosis in NAFLD subjects by analyzing liver biopsies. About 216 subjects with fatty liver were included in the study. Diagnosis of the fatty liver was made by USG. Only those patients showing fibrosis were assessed for risk factor association, in the end, they found that female patients with age $>40$ and BMI > 23 and moderate fatty grade in USG were at high risk of developing fibrosis.

A research had been conducted to examine the relationship among NAFLD and Sarcopenia in elderly patients. They (Zhai Y et al 2018. ${ }^{33}$ ) grouped the participants into Sarcopenia and Non Sarcopenia groups based on dual X-ray absorptiometry (DXA), muscle strength (grip strength) and physical performance (6m usual gait speed). They diagnosed NAFLD by USG. They found out that NAFLD was considerably less frequent in the Sarcopenia group as compared to the non Sarcopenia group $(\mathrm{P}<0.01)$. Finally, they concluded as NAFLD is not independently related to Sarcopenia.

A study had been conducted to examine the development of NAFLD in the first 3 years of antipsychotic treatment in first episode non-affective psychosis patients. In this study they selected 191 subjects, and they randomly assigned Aripiprazole to 83 subjects, Risperidone to 12 subjects, Quetiapine to 46 subjects, Ziprasidone to 50 subjects. At the intake 180 patients were anti-psychotic naive. After 3 months and then yearly for 3 years, they (Morlan- Coarasa M $\mathrm{J}$ et al $2016 .^{34}$ ) calculated the NAFLD fibrosis score, fibrosis score and FLI at baseline. Finally, through their study they concluded that there is a potential related with the development of NAFLD with schizophrenic patients receiving anti-psychotic medication.

A study had been conducted to examine the relationship associated with urine $\mathrm{pH}$ and incident NAFLD. In their (Okamura $\mathrm{T}$ et al 2018. ${ }^{35}$ ) study, they selected 11,012 individuals (5503 men and 5509 women). They divided the individuals into 3 different groups. The $\mathrm{pH}$ of urine in lowest, medium and highest group is less than or equal to 5.5, 6, >6.5 respectively. They had done cox proportional hazards models to examine the effect of urine $\mathrm{pH}$ in incident NAFLD. Finally, through their study they concluded that low urine $\mathrm{pH}$ is related to the incident NAFLD.

A longitudinal study had done to examine the association of cigarette smoking with NAFLD onset. For this study they selected 7,905 Japanese subjects. Finally, through their (Okamoto $\mathrm{M}$ et al 2018. ${ }^{36}$ study they concluded as cigarette smoking may have the ability to cause NAFLD.
A research was conducted by Xiong J. et al $2017 .{ }^{37}$ to identify the correlation involved with hepatitis $\mathrm{B}$ virus infection and NAFLD. They had done their study on hepatitis B infected individuals and uninfected control individuals. Finally, they found out that the chance of developing NAFLD is reduced in hepatitis B infected individuals than in non-infected individuals. Hence, they concluded as, the relation between hepatitis B virus infection and risk for developing NAFLD is contrary.

A study was conducted by Lin MS et al $2017 .^{38}$ to establish the relation between waist to height ratio and NAFLD in children and adolescence. For their study they selected children of age 10 to 19 years old. The exclusion was made for children those who have hepatic disease and alcohol consumption. The diagnosis was done using ultra sound reports (considered age, sex, anthropometric measurements and laboratory data). Finally, through their study they found out that, for the children who had developed NAFLD anthropometric measurements and laboratory data were higher than the normal. So, the association between waist to height ratio and NAFLD is prominent.

A research was conducted by Lapumnuay. Pol $\mathrm{K}$, et al $2018 .^{39}$ to identify the relationship between the use of inflammatory bowel disease medication and the advancement of NAFLD. They had done 7 observational studies with 1610 patients and found out that there is no occurrence of NAFLD. Finally, they concluded as there is no essential relation between inflammatory bowel disease medication and advancement of NAFLD.

A research had been carried out by Marchesini G. et al $2001 .^{40}$ suggested that the leading metabolic disease associated with NAFLD are obesity, insulin resistance and abnormal lipid metabolism. So the risk about these conditions must be considered in NAFLD patients.

The NAFLD is associated with metabolic syndrome, suggested by Misra VL. et al 2009. ${ }^{41}$ There is a high possibility of cardiovascular disorders related with NAFLD. So the patients, who are on treatment, cardiovascular risk must be considered and managed accordingly

Allard JP, et al,2002. ${ }^{42}$ suggested that NAFLD is normally seen with obesity, dyslipidemia, T2DM and other related conditions like jejunoileal bypass and gastroplasty, massive small bowel resection, rapid weight loss, certain nutritional deficiencies and bacterial over growth and extreme malnutrition. It occurs as the result of abnormal glucose and fat metabolism, unnecessary added up calories, glucose and lipids. Apart from this, drugs also play a role in disease progression.

An assessment was done by, Lavine JE, et al $2004 .^{43}$ on pediatric population about occurrence of NAFLD. Development of NAFLD is rare in children but chances of occurrence are still there in obese condition. Recent studies suggest that insulin resistance and oxidative stress plays a role in pathogenesis of NAFLD. Finally, they suggested, there is a difference between pediatric and adult fatty liver and differs in treatment.

A hypothesis is suggested by Castano-Rodriguez $\mathrm{N}$ et al $2017 .{ }^{44}$ NAFLD has become a 


\section{A Recent Research on Non Alcoholic Fatty Liver Disease}

common chronic liver disease globally. The association between gut dysbiosis and NAFLD were well established. The Helicobacter pylori infection and NAFLD are related. This may lead to increased intestinal permeability, inflammatory responses, altered carbohydrate metabolism, insulin resistance, bacterial metabolites and Helicobacter toxins. So these abnormalities must be considered in patients who are having NAFLD with Helicobacter pylori infection.

A study had been carried out by, Fucc et al $2009 .{ }^{45}$ to inspect the risk factors for NAFLD in adolescents. They enrolled 1724 adolescent students in their study. The diagnosis was made on the basis of USG findings, serum hepatitis B surface antigen (HBSAg) and anti-hepatitis C viral antibody. They concluded as obesity, ALT, HDL alone cannot be considered as an indicator but routine monitor of liver function t.est should be done in obese adolescents.

\section{E.Study report related to complications of NAFLD}

Sánchez-Jiménez BA et al $2018 .{ }^{46}$ proposed a study to assess the influence of alcohol and obesity in the development of cardiac and liver diseases. The method used in the study was a retrospective study. Subjects of the study were patients from regular follow-up. They were classified into 5 categories based on (1) healthy, (2) obesity with steatosis, (3) alcoholism with steatosis, (4) both alcoholic and NASH (5) unknown steatosis. Later patients were evaluated for alcohol consumption and cardiovascular risk. From the study, they concluded that both alcoholic and NASH had an additive outcome on the development of liver and heart diseases.

Kim GA et al 2018. ${ }^{47}$ did a research to explore the incidence rates of cancer in NAFLD and figure out the link between NAFLD and tumor development. It was a prospective study where patients were followed up for greater than 365 days. The NAFLD was detected via USG of steatosis without any other liver abnormalities/ diseases. After the prescribed period of time, out of 25,947 subjects, 8,721 patients developed NAFLD. The incidence rate of the tumor was high in patients with NAFLD than in patients exclusive of NAFLD. The disease shows firm guild with hepatocellular carcinoma, colorectal cancer in men and breast cancer in women. The study demonstrated the association of NAFLD and cancers.

Recently researchers investigated the guild of NAFLD and subclinical coronary atherosclerosis in an asymptomatic population. Coronary computed tomography angiography (CCTA) is used for the detection of Coronary atherosclerosis. 5,121 asymptomatic individuals with no history of coronary atherosclerosis were integrated in the study. USG was used to evaluate the fatty liver. NAFLD fibrosis score and the FLI were also estimated. Plaques were assessed using CCTA. From the study, Lee SB et al $2018 .{ }^{48}$ concluded that NAFLD was significantly associated with subclinical coronary atherosclerosis.

Prolonged extrahepatic aggravations of NAFLD and the evaluation of NAFLD effect in non-obese patients were studied by Kim SS et al 2018. ${ }^{49}$ It was a retrospective study includes 2,920 subjects. USG was used to diagnose NAFLD. Patients were grouped as per BMI, NAFLD, and metabolic syndrome. From the study, they understood that non-obese NAFLD subjects had a high risk of hyperglycemia than hypertension or cardiovascular disease, but in metabolically unstable non-obese subjects they had higher chances of cardiovascular disease and hypertension.

A study had been carried out by Kim S et al, $2017 .^{50}$ to examine the relationship among NAFLD with the progression of nephrolitiasis in Korean men and women. They implemented this cohort study on Korean adults, who were doing health checkup annually. The diagnosis was made using ultra sonographic reports. Finally, they concluded as NAFLD was related with nephrolithiasis, the prevalence is higher in men than in women.

The association of NAFLD with the occurrence of its progression to reflex esophagities was investigated by, Min Y W et al, $2018 .^{51}$ for their study they enrolled healthy men and women. The diagnosis was made using ultrasound on standard criteria and they found out that the incidence rate was about $33.2 \%$ and the occurrence of Reflex esophagities in the study subjects with or without NAFLD were $9.6 \%$ and $13.8 \%$ respectively. Finally, they concluded as NAFLD was not considerately correlated with progression of reflex esophagities but it may also link with BMI.

A study was conducted by Kanwal F et al, 2018. ${ }^{52}$ to understand the possibility of development of hepatocellular cancer in NAFLD patients. Patients diagnosed with NAFLD were included in the study and check out them till hepatocellular cancer development or death. They also included a control group for their study. In their study they compared 2, 96707 NAFLD patients with the 2, 96707 control population and they found out that there is a higher probability of progress it to hepatocellular cancer in NAFLD patients.

\section{F.Study reports related to diagnosis of NAFLD}

Mouzaki M et al 2018. ${ }^{53}$ performed a research to evaluate disease advancement by using paired magnetic resonance imaging (MRI) measurement. 65 patients with NAFLD who underwent recurrent MRI studies were included in the study. The methodology used was a retrospective cohort study. Statistical analysis used in the study includes the Pearson or Spearman correlation. Liver fat fraction (FF) was analyzed with the help of a mixed replica analysis. In the results, they obtained a feeble positive relationship between fat fraction change and ALT change but there were no statistically considerable changes in High-Density Lipoprotein (HDL) insulin, glucose and Low-Density Lipoprotein (LDL). From the study, they concluded that MRI based studies were complementary in the evaluation of NAFLD.

\section{G. Study reports related to treatment of NAFLD}

S. Spahis et al 2018. ${ }^{54}$ demonstrated a study that compares the 2 levels of severity of NAFLD in obese adult patients with related to hyperlipidemia, liver enzymes (AST, ALT) metabolic derangements, oxidative stress, tenderness and to calculate the potency and safety reaction to n-3 polyunsaturated fatty acid treatment. The study includes 20 adult male volunteers of French- Canadian basis with the disease. They are further classified into moderately and severely affected groups according to their level of liver enzymes, USG, FTI. The severely affected NAFLD volunteers were advised to consume $2 \mathrm{~g}$ of fish oil that consists of omega-3 fatty acids for 
6 months. The polyunsaturated fatty acid improved their lipid profile by the end of 6 months. From the study, they concluded that omega- 3 fatty acid has a beneficial role in the treatment of NAFLD. A study was conducted to explore the effectiveness of vitamin D supplementations in the therapy of subjects with NAFLD. The patients were randomly allowed to take a placebo (one weekly), cholecalciferol/ vitamin D3 (per weekly) and calcitriol (per day) for 3 months. After 12 weeks of treatment, serum alkaline phosphatase levels were greatly reduced from baseline in cholecalciferol and calcitriol-treated patients.

Gamma-glutamyl transferase levels were also reduced in patients treated with cholecalciferol after 12 weeks. From the study, Dabbaghmanesh $\mathrm{MH}$ et al $2018 .^{55}$ concluded that vitamin D supplementation is effective in the treatment of NAFLD. A research was conducted to understand the discrepancy between fatty acid content in patients with NAFLD and normal healthy individuals. The changes in the lipid content in both the groups were measured as Weighted Mean Differences (WMD) and the variations in the liver enzymes, fat content, and glucose was evaluated after the introduction of an intervention. The results of the research showed that the docosahexaenoic acid content was high in healthy individuals than patients. Guo XF et al 2018. ${ }^{56}$ concluded that docosahexaenoic acid has a beneficial part in the treatment of NAFLD.

The study related to the effectiveness of olive oil on cardiometabolic markers and severity of fatty liver was conducted by Rezaei S et al 2018. ${ }^{57}$ It was a double-blind, randomized control trial involving 66 patients with NAFLD. Patients were alienated into 2 categories and advised to consume either olive oil or sunflower oil each $20 \mathrm{~g} /$ day for 1 month. A hypocaloric diet was strictly followed in each group. These parameters were assessed; Fatty liver grade, serum glutamic oxaloacetic transaminase (SGOT), serum glutamic pyruvic transaminase (SGPT), weight, height, blood pressure (BP), serum lipids, glucose, insulin, malondialdehyde, antioxidant capacity, and IL-6. The results of the study showed that sunflower oil greatly decreased the SGPT and SGOT where olive oil only decreased SGOT. Sunflower oil also diminished fat-free mass and skeleton mass while serum triglycerides and fat mass were cut down by oil. From the study, they concluded that olive oil may mitigate the fatty liver harshness and is not dependent on cardiometabolic risk factors.

A study was conducted by Amanat $\mathrm{S}$ et al $2018 .{ }^{58}$ to understand the valuable response of genistein supplementation on a NAFLD. It was a double-blind, randomized control trial and the subjects were recommended to consume genestein supplements $250 \mathrm{mg}$ or placebo daily for 8 weeks. Subjects weight, height and biochemical parameters were assessed before and after the introduction of the therapeutic drug. Subjects were also advocated to pursue a balanced diet and restricted physical activities. Investigators concluded that, interventional group had decrease point of insulin, body fat percentage, triglycerides, serum malondialdehyde (MDA), tumour necrosis factor- $\alpha$ (TNF- $\alpha$ ) and IL-6. From the study, they concluded that oral supplementation of genistein $250 \mathrm{mg}$ for 8 -weeks had beneficial effects in NAFLD.
Therapeutic effect of Spirulina on an animal replica of the high-fat-diet-causing non-alcoholic fatty liver (HFD-NAFL) was investigated by Oriquat GA et al 2018. ${ }^{59}$ Animals used in the present experiments were Wistar rats each weighing $120-150 \mathrm{~g}$. The sample size used in the study was 50 . The rats were classified into 3 groups (1) NAFL rats, (2) NAFL treated group, (3) a control group. NAFL treated groups received Spirulina suspension orally in the dose of 100, 200, $500 \mathrm{mg} / \mathrm{kg}$ and untreated groups with saline orally for 30 days. Later, the animals were sacrificed after collecting their fasting blood samples by cardiac puncture and centrifugation. Livers were also gathered and assessed for molecular, histological and biochemical parameters. At the end, they observed that NAFL rats treated with Spirulina had low levels of fasting blood glucose, liver enzymes compared to NAFL untreated and control groups.

Kim SS et al $2018 .^{60}$ conducted a study to set up the efficacy of Oxyresveratrol in the treatment of NAFLD. Oxyresveratrol is an herbal medicine and a natural polyphenolic compound which is present mostly in white mulberry. The compound is a powerful inhibitor of hepatic, hyperglycemic, microbial, hypertensive, allergic and neurologic ailments. The experiment was performed in human hepatocyte-derived cell lines and 6 male mice. Standard conditions which are required were strictly followed during the research. Statistical analyses used to evaluate the results were the One-way ANOVA, mean, standard deviation, and Mann-Whitney test (U test). From the study, they concluded that oxyresveratrol had the potential in the treatment of hepatic steatosis by inhibiting liver $\mathrm{X}$ receptor $\alpha-256(\mathrm{LXR} \alpha-256)$ sterol regulatory element binding protein 1c (SREBP-1c) mediated lipogenic gene induction through the liver kinase B1 (LKB1) /adenosine monophospate activated protein kinase (AMPK) pathway.

Recently researchers developed a FGF19 analogue NGM282 that can be used in the treatment of NASH. Harrison SA et al $2018 .^{61}$ conducted a study to assess the effectiveness of NGM282 in NASH. Subjects involved in the study were aged 18-75 years and those patients whom NASH was biopsy-proven were involved in the study. Eligibility criteria included (1) NAFLD activity score greater than 4, (2) fibrosis stage $1-3,(3)$ at least $8 \%$ fat content in the liver. It was a randomized, double-blind, placebo-controlled, two-phase study. Subjects were randomly selected and advised to take either the test drug ( $3 \mathrm{mg}$ or $6 \mathrm{mg}$ ) or placebo. The patient who responded to the treatment shows a $5 \%$ decrease in the liver fat content. From the study, they established that NGM282 shows a significant and rapid decline in the liver fat without any serious adverse effects.

Effect of dioscin in NAFLD was studied by Yao $\mathrm{H}$ et al $2018{ }^{62} 50$ male Wistar rats $(180-220 \mathrm{~g})$ and male mice $(18-22 \mathrm{~g})$ were enrolled in the study. The animals were erratically alienated into 5 groups (1) control group, (2) replica group, (3) high dose dioscin group, (4) medium dose dioscin group, (5) low dose dioscin group. Blood samples were obtained from rats and mice of each group for evaluating the biochemistry and later tissues are also assessed for histopathological studies. GraphPad Prism 5.0 software was used for statistical 


\section{A Recent Research on Non Alcoholic Fatty Liver Disease}

analysis. From the study, they came to know that the dioscin meliorate lipid accumulation symptoms as well as hepatic and serum biomarkers in NAFLD. Further studies in the topic revealed that dioscin rarefy the lipid metabolism via modifying Silent mating type information regulation 2 homolog (SIRT1) /AMPK signal pathway.

Recent researches came forward with a hypothesis that the ammonia can be used in the management of NASH. This hypothesis was based upon 4 pieces of evidence (1) NASH induced rats lowers the Ornithine carbamoyltransferase (OTC) gene and protein gene expression results in hyperammonemia. This NASH can be reversed and the OTC genes can be regenerated if the fat diet was converted to normal eating habits.

(2) In another study lowering the level of bacterial translocation reduces OTC gene expression and NASH (3) NASH affected humans show reduced urea cycle enzymes and OTC gene expression. (4) Reduced urea cycle enzymes result in decreased ammonia clearance, on such patients there will be elevated plasma ammonia concentrations and ends in neuropsychiatric disturbances. This hypothesis review of ammonia in NASH by Thomsen KL et al $2018 .{ }^{63}$ concludes that ammonia lowering drugs can help in reducing disease advancement in NASH.

Ebrahimi $\mathrm{S}$ et al $2018 .{ }^{64}$ performed a study to assess the effect of fasting in Ramadan season on adipokines in NAFLD patients. The research was performed in 83 NAFLD subjects, 42 patients informed that they would fast in the Ramadan. 41 subjects were taken as control who advised not to fast during the month of Ramadan. From both groups, anthropometric parameters were measured before and after Ramadan. Enzyme linked immune sorbent assay (ELISA) kit was used for measuring serum adipokines including vaspin and omentin-1. At the end of the study, investigators found that there were reduced mean hip circumference and BMI and notable alterations in serum vaspin and omentin-1 in patients who fasted during the month of Ramadan. The study concluded that fasting during the month of Ramadan leads to lower the levels of adipokines.

McCarty TR et al 2018. ${ }^{65}$ performed a research to assess the interrelation among bariatric surgery and NAFLD clinical outcomes in hospitalized patients. The study was conducted for a period of 8 years. Inclusion criteria involved patients with NAFLD and co-morbid obesity. Patients with a history of bariatric surgery were also included. Statistical analysis used in the study was Poisson regression and incidence risk ratio for clinical aftereffects of patients with the history of bariatric surgery was compared with the subjects without surgery. A total of 45,462 patients had a verdict of NAFLD and obesity at the point of discharge. Among that 18,618 had the history of bariatric surgery. There was a decline in bariatric surgeries during the period of 2004 - 2012. The patient who underwent bariatric surgery had less incidence of mortality, cirrhosis, myocardial infarction, renal failures and stroke. So the researchers came to an end that bariatric surgery was linked to the low occurrence of morbidity and mortality in NAFLD patients.

Simple fatty steatosis could progress into NASH when there is an endothelial dysfunction caused by oxidative stress. Antioxidants such as polyphenols can diminish oxidative stress and regenerate the endothelial function by suppressing
NOX2. A study was conducted by Loffredo L et al $2018 .{ }^{66}$ to evaluate the oxidative stress and endothelial impairment in fatty liver disease and NASH and the effect of dark chocolate on an endothelial mechanism in patients affected with NASH. It was a crossover study involving 19 subjects with NASH. The subjects were advised to take $40 \mathrm{~g}$ of dark chocolate, cocoa for 14 days (>85\% high cocoa content) vs. milk chocolate, cocoa $(<35 \%$ low cocoa content). The results obtained were compared to the control group. In the results, the researchers obtained that the group treated with dark chocolate (high content of cocoa phenols) had improved endothelial function by suppressing NOX2.

Zhang $\mathrm{J}$ et al $2018 .^{67}$ conducted a study to investigate the hepatoprotective effects of baicalin in MCD diet-induced NASH. Baicalin was known to exhibit anti-inflammatory and antioxidant property to various hepatic diseases. The study was conducted on mice fed with MCD for 4 weeks. Simultaneous treatment with baicalin or without baicalin was given for 4 weeks. Then the liver tissues were assessed. Parameters of the liver were assessed by biochemistry and ELISA. In the end the investigators found that baicalin treatment prevented (1) liver fibrosis (2) MCD induce hepatic lipid accumulation (3) hepatic inflammation. The study concluded that baicalin played a crucial role in the treatment of NASH.

A study had been carried out by Wong V W et al, 2018. ${ }^{68}$ to analyze the impact of life style changes in non-obese patients with NAFLD. For this study they enrolled 154 NAFLD patients and they were trained for their life style changes. They found out that life style modification has a major role in treating NAFLD in both obese and non-obese patients.

The impact of rifaximin in NAFLD patients were examined by Abdel-Razik A, et al, 2018. ${ }^{69}$ They enrolled 50 individuals in their study and grouped as rifaximin and placebo group equally. After duration of 6 months they observed a reduction of ALT and AST but no effect in lipid panel. They concluded as rifaximin therapy is efficacious in NASH patients through enhancement of insulin resistance.

A study conducted to understand the clinical outcome of probiotics in the management of NAFLD, Wang W, et $a l, 2018 .^{70}$ This study enrolled 200 individuals and divided them into 4 groups. The first group was controlled group and the other 3 were combined treatment group. For the controlled group they gave polyene phosphatidy choline capsule, and for the combined group they gave live combine Bifidobacterium lactobacillus and Enterococus powder and two live combined Bacillus subtillis and Enterococcus and probiotics respectively. The duration of the study was about 1 month. For the assessment of the outcome of the study the following parameters were examined which includes, total cholesterol, HDL, LDL, ALT, AST and serum TNF- $\alpha$. The faeces samples were regularly checked and liver ultra sound scan were done. They found out blood glucose level, TNF- $\alpha$ decreased but there was no effect on HDL-Cholesterol level. All group were benefited from treatment, finally they concluded as probiotics are effective in NAFLD patients and it prevents further hepatic damage.

York LW et al, 2009. ${ }^{71}$ suggested that NAFLD is related with metabolic syndrome, insulin 
resistance and cardiovascular disorders. Diet plays a major role in management of NAFLD. Low carbohydrate diet is beneficial in management and prevention of NAFLD.

An analysis done by LA Adams, et al, 2005. ${ }^{72}$ suggested that the pathogenesis involved in steatosis and cellular injury is thought to be related to insulin resistance and oxidative stress, so the management can be done by identifying and treating the metabolic risk factors, improving insulin sensitivity and increasing antioxidant defenses in the liver along with weight management and life style modification. A study had been conducted by Osaka T, et al, $2018 .^{73}$ to understand the benefit of exercise in NAFLD. They enrolled 1,010 individuals ( 860 men and 150 women) in their study. USG images were used to diagnose NAFLD.

A 10 year follow up was done and found out that the reduction of NAFLD in men and women were $46 \%$ and $48.7 \%$ respectively. Finally, they concluded as exercise at least once per week is concerned with the subsidence of NAFLD in men.

A study had been carried out by Mazzella N. et al, $2014 .^{74}$ to make out the effectiveness of controlled trials in the treatment of NAFLD. Many drugs with diverse mechanisms were checked its effectiveness in series of events from fatty liver to fibrosis, cirrhosis, and hepatocellular cancer. They suggested treatment should be done based on age and other patient related factors to prevent the disease progression.

Corrado RL, et al, $2014 .^{75}$ suggested that even though many advancements were done in the treatment of NAFLD and $\mathrm{NASH}$, many challenges were faced in modifying life styles. For the proper diagnosis and management of NAFLD, the co-morbid conditions, exercise tolerance and patient adherence with therapy must be considered to prevent the further disease progression.

The hypothesis done by Fleet SE et al, $2017 .{ }^{76}$ suggested that, NAFLD, refers to a variety of diseases and its incidence increases due to obesity. Hepatocyte lipid accumulation is considered as the cause for metabolic and inflammatory events. Lifestyle modification is important in treating NAFLD in obese children. Treatment with vitamin $\mathrm{E}$ is recommended. Many adult medications efficiency must be checked before initiating treatment with these drugs in children. Biopsy findings are needed for improving the treatment in future.

A research had been carried out by, Trappoliere. M, et al, $2005 .^{77}$ to understand the treatment procedures in NAFLD. The treatment started with an aim to prevent its progression to liver cirrhosis and hepatocellular carcinoma. The management mainly focused on underlying conditions like obesity, diabetes mellitus and hyperlipidemia. The insulin resistance can be managed using two classes of drugs, biguanides and thiazolidinediones. hepato protective drugs are also useful in management of NAFLD.

A study had been carried out in NAFLD patients treated with omega 3 poly unsaturated fatty acids to assess the changes in erythrocyte docosahexaenoic acid enrichment, a surrogate marker for tissue enrichment \{Hodson L, et al, $2017 .^{78}$. They found out that individuals who showed docosahexaenoic acid enrichment $>2 \%$ have changes in hepatic fatty acid metabolism and insulin sensitivity which leads to the reduction of hepatic fat content.

A hypothesis had been carried out to assess the effectiveness of bile acid in NAFLD or NASH \{Orlando R, 2007. ${ }^{79}$ \}. Based on their suggestion on 4 randomized clinical trials of 279 patients, they found out that there is no significant effect on the liver function test with treatment of ursedeoxycholic acid. The adverse event occurred with this therapy were non-specific and less prevalent.

As per the case reported in a patient who was treated with leuprorelin acetate for 32 months, NAFLD with focal lesion at intravenous-hepatic segment was encountered. It appears to be more severe $\left\{\right.$ Gabbi C et al, 2008. ${ }^{80}$ \}. In addition to this the patient also diagnosed with hypotestosteronemia and metabolic syndrome including insulin resistance. So, regular monitor should be required in patients who are in treatment with leuporelin acetate.

As per the findings made by Rafiq $\mathrm{N}$ et al, $2008{ }^{81}$ suggested that NAFLD is linked with obesity, metabolic syndrome and insulin resistance. So, the management mainly focused on weight reduction, insulin sensitizers, anti-hyperlipidemias and antioxidants. Bariatric surgery done for weight reduction also has its own role in treatment. So, weight reduction is beneficial in the treatment of NAFLD.

As per the case reported, studies were done by, Verrotti. A et al, 2009. ${ }^{82}$ to find out the hyperinsulinemia with insulin resistance and increased weight associated with long term valproate therapy. They found that after the withdrawal of therapy, BMI, metabolic and endocrine functions becomes normal. So, the patients should be monitored for NAFLD development on long term use of valproate.

A study had been carried out by, Abd El Kander SMI, et al, $2016{ }^{83}$ to identify the bio chemical constraints responsible for weight loss in NASH patients. For their study they enrolled 100 obese patients of age between 35-50 years, having BMI $30-35 \mathrm{Kg} / \mathrm{m}^{2}$ and they were separated into 2 groups. One group trained with exercise and diet and others were having no treatment measures. They found out that leptin, TNF- $\alpha$, IL-6, interleukin -8 (IL8), ALT, AST, LDL, and BMI decreased in first group and HDL was increased. No changes were identified in control group. They concluded as weight reduction is beneficial in management of NASH.

A study had been carried out by, Sofer EI, et al, $2016 .{ }^{84}$ to evaluate the effect of metformin therapy on circulating osteoprotegerin in patients with NAFLD. For their study they enrolled 63 patients and they found out that the patients treated with metformin, osteoprotegerin and ALP decreased and CRP and ALT decreased during 4 months of therapy. They concluded as metformin treatment is beneficial in treating osteoprotegerin levels in NAFLD patients.

The NAFLD and NASH is becoming a common liver disease, so its treatment is challenging and should be concentrated on different pathways for the treatment. Many metabolites and natural supplements were used \{Ilan Y, et al, 2018. ${ }^{85}$ \} The manufacturing, quality control, clinical development should be assessed, if properly managed these compounds are having the potentiality to meet 


\section{A Recent Research on Non Alcoholic Fatty Liver Disease}

the challenges associated with NAFLD therapy.

\section{CONCLUSION}

NAFLD is becoming a common liver disease as its estimated prevalence is about $20-30 \%$ in Western population. ${ }^{86}$ The most common co-morbidities associated with NAFLD include obesity and metabolic syndrome. ${ }^{87}$ There is a disease progression from NAFLD to hepatocellular carcinoma. The main goal involved in management of NAFLD is to reduce the histological features and insulin resistance and normalize the liver enzyme level. ${ }^{88}$ Proper diagnosis is important for the management of the disease. Intakes of fatty foods, lack of exercise are some of the contributing factors which lead to NAFLD. The main goal in management of NAFLD is to prevent the complications associated with the disease. Special importance must be given to avoid the cardiovascular risk.

Bariatric surgery can be done in patients who fails to respond to the current plan of treatment and it must be done before the patient become cirrhotic. ${ }^{89}$ NAFLD is a complex condition so the management of NAFLD varies from person to person depending upon their conditions. Exercise and weight loss is most effective in management of NAFLD. Along with medication life style modification also has a major role in managing NAFLD. Regular exercise, controlled body weight, good dietary intake, and less or no alcohol intake are beneficial in preventing NAFLD Fig 2.

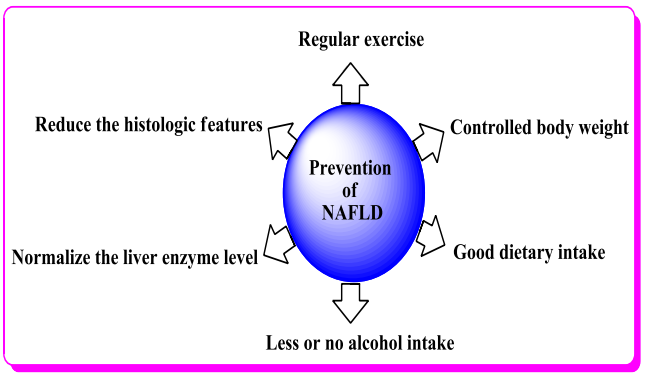

Figure 2: Management of NAFLD

\section{REFERENCES}

1. Farrell GC, Larter CZ. Nonalcoholic fatty liver disease: from steatosis to cirrhosis. Hepatology 2006; 43: S99-112.

2. Chalasani, Naga, Zobair Younossi, Joel E. Lavine, Anna Mae Diehl, Elizabeth M. Brunt, Kenneth Cusi, et al. The diagnosis and management of non-alcoholic fatty liver disease: Practice Guideline by the American Association for the Study of Liver Diseases, American College of Gastroenterology, and the American Gastroenterological Association. Hepatology 2012; 55: 2005-2023.

3. Benedict M, Zhang X. Non-alcoholic fatty liver disease: An expanded review. World J Hepatol 2017; 9: 715.

4. Marchesini G, Brizi M, Bianchi G, Tomassetti S, Bugianesi E, Lenzi M, et al. Nonalcoholic fatty liver disease: a feature of the metabolic syndrome. Diabetes 2001; 50: 1844-50.

5. Paschos P, Paletas K. Non alcoholic fatty liver disease and metabolic syndrome. Hippokratia 2009; 13: 9.

6. Tolman KG, Dalpiaz AS. Treatment of non-alcoholic fatty liver disease. Ther Clin Risk Manag 2007; 3:1153

7. De Chiara, Francesco, Sara Heebøll, Giusi Marrone, Carmina Montoliu, Stephen Hamilton-Dutoit, et al. Urea cycle dysregulation in non-alcoholic fatty liver disease. J Hepatol 2018; 69: 905-915.

8. Bae CR, Hino J, Hosoda H, Miyazato M, Kangawa K. C-type natriuretic peptide (CNP) in endothelial cells attenuates hepatic fibrosis and inflammation in non-alcoholic steatohepatitis. Life Sci 2018; 15: 349-56

9. Xu Y, Lu LL, Liu SS, Du SX, Zhu HL, Dong QJ, et al. Apolipoprotein A5 gene polymorphisms are associated with non-alcoholic fatty liver disease. HBPD INT 2018; 17: 214-9.

10. Matsumoto M, Zhang J, Zhang X, Liu J, Jiang JX, Yamaguchi K, et al The NOX1 isoform of NADPH oxidase is involved in dysfunction of liver sinusoids in nonalcoholic fatty liver disease. Free Radic Biol Med 2018; 115: 412-20

11. Yamada T, Murata D, Adachi Y, Itoh K, Kameoka S, Igarashi A, et al. Mitochondrial Stasis Reveals p62-Mediated Ubiquitination in Parkin-Independent Mitophagy and Mitigates Nonalcoholic Fatty Liver Disease. Cell metab 2018; 28: 588-604.

12. Borai IH, Shaker YM, Kamal MM, Ezzat WM, Ashour E, Afify M, et al Association of nonalcoholic fatty liver disease grades with the plasma cell antigen-1 (PC-1) gene polymorphism. EJMGH 2018; 19: 241-6.

13. Zhou W, Wang Y, Wu Y, Yang J, Xu L, Yang Y. Serum CTRP3 level is inversely associated with nonalcoholic fatty liver disease: A 3-y longitudinal study. Clin Chim Acta 2018; 479: 79-83.

14. González-Rodríguez Á, Valdecantos MP, Rada P, Addante A, Barahona $\mathrm{I}$, Rey E, et al. Dual role of protein tyrosine phosphatase $1 \mathrm{~B}$ in the progression and reversion of non-alcoholic steatohepatitis. Mol metab 2018; 7: 132-46.

15. Seko Y, Yamaguchi K, Mizuno N, Okuda K, Takemura M, Taketani H, et al. Combination of PNPLA3 and TLL1 polymorphism can predict advanced fibrosis in Japanese patients with nonalcoholic fatty liver disease. Gastroenterol 2018; 53: 438-48

16. Vassilatou E, Lafoyianni S, Vassiliadi DA, Ioannidis D, Paschou SA Mizamtsidi M, et al. Visceral adiposity index for the diagnosis of nonalcoholic fatty liver disease in premenopausal women with and without polycystic ovary syndrome. Maturitas 2018; 16: 1-7.

17. Ikarashi Y, Kodama K, Taniai M, Hashimoto E, Tokushige K. The Clinical Difference in the Platelet Counts between Liver Cirrhosis with Nonalcoholic Fatty Liver Disease and Hepatitis C Virus J. Intern. Med. 2018; 57: 1065-70.

18. Dong S, Zhan ZY, Cao HY, Wu C, Bian YQ, Li JY, et al. Urinary metabolomics analysis identifies key biomarkers of different stages of nonalcoholic fatty liver disease. World J Gastroenterol 2017; 23: 2771.

19. Hagström H, Nasr P, Bottai M, Ekstedt M, Kechagias S, Hultcrantz R, et al. Elevated serum ferritin is associated with increased mortality in non-alcoholic fatty liver disease after 16 years of follow-up. Liver Int 2016; 36: 1688-95

20. Dai W, Ye L, Liu A, Wen SW, Deng J, Wu X, et al. Prevalence of nonalcoholic fatty liver disease in patients with type 2 diabetes mellitus: A meta-analysis. Medicine 2017; 96.

21. Bellentani S, Scaglioni F, Marino M, Bedogni G. Epidemiology of non-alcoholic fatty liver disease. Dig Dis 2010; 28: 155-61.

22. Van den Berg EH, Gruppen EG, Ebtehaj S, Bakker SJ, Tietge UJ, Dullaart RP. Cholesterol efflux capacity is impaired in subjects with an elevated Fatty Liver Index, a proxy of non-alcoholic fatty liver disease. Atherosclerosis 2018; 277: 21-7.

23. Li TT, Wang AP, Lu JX, Chen MY, Zhao CC, Tang ZH, et al. Prevalence and clinical characteristics of NAFLD in newly diagnosed patients with ketosis-onset diabetes. Diabetes \& metab 2018.

24. Okamura T, Hashimoto Y, Hamaguchi M, Obora A, Kojima T, Fukui M. Low urine $\mathrm{pH}$ is a risk for non-alcoholic fatty liver disease: A population-based longitudinal study. Clin Res Hepatol Gastroenterol 2018.

25. Afolabi BI, Ibitoye BO, Ikem RT, Omisore AD, Idowu BM, Soyoye DO The Relationship Between Glycaemic Control and NAFLD in Nigerian Type 2 Diabetic Patients. J Natl Med Assoc 2018; 110: 256-64.

26. .Dowla S, Pendergrass M, Bolding M, Gower B, Fontaine K, Ashraf A, et al. Effectiveness of a carbohydrate restricted diet to treat NAFLD in adolescents with obesity: Trial design and methodology. Contemp Clin Trials 2018; 68: 95-101.

27. Wang L, Li M, Zhao Z, Xu M, Lu J, Wang T, et al. Ideal cardiovascular health is inversely associated with non-alcoholic fatty liver disease: a prospective analysis. Am J Med 2018; 131: 1515-e1.

28. Chou YT, Cheng HJ, Wu JS, Yang YC, Chou CY, Chang CJ, et al. The association of sleep duration and sleep quality with NAFLD in a Taiwanese population. Obes Res Clin Pract 2018;

29. Chang HT, Pan HJ, Lee CH. Prevention of Tamoxifen-related Nonalcoholic Fatty Liver Disease in Breast Cancer Patients. Clin. breast cancer 2018; 18: 677-85

30. Ajmera V, Belt P, Wilson LA, Gill RM, Loomba R, Kleiner DE, et al Among patients with nonalcoholic fatty liver disease, modest alcohol use is associated with less improvement in histologic steatosis and steatohepatitis. Clin Gastroenterol Hepatol 2018.

31. Liu P, Tang Y, Guo X, Zhu X, He M, Yuan J, et al. Bidirectional association between nonalcoholic fatty liver disease and hypertension from the Dongfeng-Tongji cohort study. J Am Soc Hypertens 2018.

32. Pattnaik K, Bhuyan P, Singh A, Singh SP, Nath P, Kar S, et al. Biopsy Proven Fibrosis in Non-Alcoholic Fatty Live

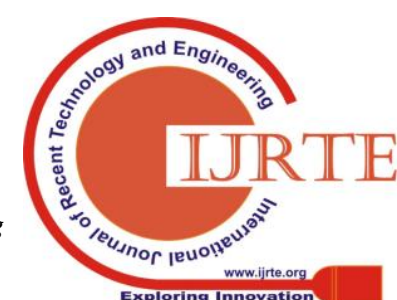


Disease: An Analysis of Risk Factors. J Clin Exp Hepatol 2018.

33. Zhai Y, Xiao Q, Miao J. The Relationship between NAFLD and Sarcopenia in Elderly Patients. Can J Gastroenterol Hepatol 2018.

34. Morlán-Coarasa MJ, Arias-Loste MT, de la Foz VO, Martínez-García O, Alonso-Martín C, Crespo J, et al. Incidence of NAFLD and metabolic dysfunction in first episode schizophrenia and related psychotic disorders: a 3-year prospective randomized interventional study. Psychopharmacology 2016; 233: 3947-52.

35. Okamura T, Hashimoto Y, Hamaguchi M, Obora A, Kojima T, Fukui M. Low urine $\mathrm{pH}$ is a risk for non-alcoholic fatty liver disease: A population-based longitudinal study. Clin Res Hepatol Gastroenterol 2018.

36. Okamoto M, Miyake T, Kitai K, Furukawa S, Yamamoto S, Senba H, et al. Cigarette smoking is a risk factor for the onset of fatty liver disease in nondrinkers: A longitudinal cohort study. PloS one 2018; 13: e0195147.

37. Xiong J, Zhang H, Wang Y, Wang A, Bian J, Huang H, et al. Hepatitis B virus infection and the risk of nonalcoholic fatty liver disease: a meta-analysis. Oncotarget 2017; 8: 107295.

38. Lin MS, Lin TH, Guo SE, Tsai MH, Chiang MS, Huang TJ, et al. Waist-to-height ratio is a useful index for nonalcoholic fatty liver disease in children and adolescents: a secondary data analysis. BMC public health $2017 ; 17: 851$

39. Lapumnuaypol K, Kanjanahattakij N, Pisarcik D, Thongprayoon C, Wijarnpreecha K, Cheungpasitporn W. Effects of inflammatory bowel disease treatment on the risk of nonalcoholic fatty liver disease: a meta-analysis. Eur J Gastroenterol Hepatol 2018; 30: 854-60.

40. Marchesini G, Brizi M, Bianchi G, Tomassetti S, Bugianesi E, Lenzi M, et al. Nonalcoholic fatty liver disease: a feature of the metabolic syndrome. Diabetes 2001; 50: 1844-50.

41. Misra VL, Khashab M, Chalasani N. Nonalcoholic fatty liver disease and cardiovascular risk. Curr Gastroenterol Rep 2009; 11: 50.

42. Allard JP. Other disease associations with NAFLD. Best Pract Res Clin Gastroenterol 2002; 16: 783-95.

43. Lavine JE, Schwimmer JB. Nonalcoholic fatty liver disease in the pediatric population. Clin Liver Dis 2004; 8: $549-58$

44. Castaño-Rodríguez N, Mitchell HM, Kaakoush NO. NAFLD, Helicobacter species and the intestinal microbiome. Best Pract Res Clin Gastroenterol 2017; 31: 657-68.

45. Fu CC, Chen MC, Li YM, Liu TT, Wang LY. The risk factors for ultrasound-diagnosed NAFLD among adolescents. Ann Acad Med Singapore 2009; 38: 15.

46. Sánchez-Jiménez BA, Brizuela-Alcántara D, Ramos-Ostos $\mathrm{MH}$ Alva-López LF, Uribe-Esquivel M, Chávez-Tapia NC. Both alcoholic and non-alcoholic steatohepatitis association with cardiovascular risk and liver fibrosis. Alcohol 2018; 69: 63-7.

47. Kim GA, Lee HC, Choe J, Kim MJ, Lee MJ, Chang HS et al. Association between NAFLD and cancer incidence rate. J Hepatol. 2018; 68:140-6.

48. Lee SB, Park GM, Lee JY, Lee BU, Park JH, Kim BG, Association between NAFLDand subclinical coronary atherosclerosis: An observational cohort study. J Hepatol. 2018; 68:1018-24.

49. Kim SS, Cho HJ, Kim HJ, Kang DR, Berry JR, Kim JH et al. Nonalcoholic fatty liver disease as a sentinel marker for the development of diabetes mellitus in non-obese subjects. Dig Liver Dis. 2018 ; 50:370-7.

50. Kim S, Chang Y, Sung E, Kim CH, Yun KE, Jung HS, Shin H, Ryu S. NAFLDand the development of nephrolithiasis: A cohort study. PloS one. 2017;12: e0184506.

51. Min YW, Kim Y, Gwak GY, Gu S, Kang D, Cho SJ, et al. Non-alcoholic fatty liver disease and the development of reflux esophagitis: A cohort study. J Gastroenterol Hepatol. 2018. 33:1053-8.

52. Kanwal F, Kramer JR, Mapakshi S, Natarajan Y, Chayanupatkul M, Richardson PA,

et al. Risk of Hepatocellular Cancer in Patient with Non-alcoholic Fatty Liver Disease. Gastroenterol. 2018.

53. Mouzaki M, Trout AT, Arce-Clachar AC, Bramlage K, Kuhnell P, Dillman JR, Xanthakos S. Assessment of Nonalcoholic Fatty Liver Disease Progression in Children Using Magnetic Resonance Imaging. $J$ Pediatr.2018.

54. Spahis S, Alvarez F, Ahmed N, Dubois J, Jalbout R, Paganelli M. et al NAFLD severity and metabolic complications in obese children: impact of omega-3 fatty acids. J Nutr. Biochem. 2018:58:28-36.

55. Dabbaghmanesh MH, Danafar F, Eshraghian A, Omrani GR. Vitamin D supplementation for the treatment of non-alcoholic fatty liver disease: A randomized double-blind placebo controlled trial. Diabetes Metab Syndr Clin Res Rev. 2018

56. Guo XF, Yang B, Tang J, Li D. Fatty acid and non-alcoholic fatty liver disease: Meta-analyses of case-control and randomized controlled trials. Clin Nutr. 2018;37:113-22

57. Rezaei S, Akhlaghi M, Sasani MR, Boldaji RB. Olive oil lessened fatty liver severity independent of cardiometabolic correction in patients with non-alcoholic fatty liver disease: A randomized clinical trial. Nutr.J. 2019 Jan ;57:154-61.

58. Amanat S, Eftekhari MH, Fararouei M, Lankarani KB, Massoumi SJ. Genistein supplementation improves insulin resistance and inflammatory state in non-alcoholic fatty liver patients: A randomized, controlled trial. Clin Nutr. 2018;37:1210-5.

59. Oriquat GA. Therapeutic effects of Spirulina against experimentally-induced non-alcoholic fatty liver in rats may involve miR-21,-34a and-122. Meta Gene.2018.

60. Kim SS, Cho HJ, Kim HJ, Kang DR, Berry JR, Kim JH.et al Nonalcoholic fatty liver disease as a sentinel marker for the development of diabetes mellitus in non-obese subjects. Dig Liver Dis. 2018;50:370-7.

61. Harrison SA, Rinella ME, Abdelmalek MF, Trotter JF, Paredes AH Arnold HL,et al. NGM282 for treatment of non-alcoholic steatohepatitis: a multicentre, randomised, double-blind, placebo-controlled, phase 2 trial. Lancet. 2018;391:1174-85.

62. Yao H, Tao X, Xu L, Qi Y, Yin L, Han X, et al. NAFLD through adjusting lipid metabolism via SIRT1/AMPK signaling pathway. Pharmacol Res. 2018;131:51-60.

63. Thomsen KL, De Chiara F, Rombouts K, Vilstrup H, Andreola F, Mookerjee RP, Jalan R. Ammonia: A novel target for the treatment of non-alcoholic steatohepatitis. Med Hypotheses. 2018;113:91-7.

64. Ebrahimi S, Gargari BP, Izadi A, Imani B, Asjodi F.The effects of Ramadan fasting on serum concentrations of vaspin and omentin-1 in patients with nonalcoholic fatty liver disease. Eur $J$ In tegr Med. 2018;19:110-4.

65. McCarty TR, Echouffo-Tcheugui JB, Lange A, Haque L, Njei B. Impact of bariatric surgery on outcomes of patients with nonalcoholic fatty liver disease: a nationwide inpatient sample analysis, 2004-2012. Surg Obes Relat Dis. 2018;14:74-80.

66. Loffredo L, Baratta F, Ludovica P, Battaglia S, Carnevale R, Nocella C, et al. Effects of dark chocolate on endothelial function in patients with non-alcoholic steatohepatitis. $\mathrm{Nu}$ tr, Me tab and Cardiovasc Dis. 2018;28:143-9.

67. Zhang J, Zhang H, Deng X, Zhang N, Liu B, Xin S,et al. Baicalin attenuates non-alcoholic steatohepatitis by suppressing key regulators of lipid metabolism, inflammation and fibrosis in mice. Life Sci J. 2018;192:46-54

68. Wong VW, Wong GL, Chan RS, Shu SS, Cheung BH, Li LS,et al. Beneficial effects of lifestyle intervention in non-obese patients with non-alcoholic fatty liver disease. J Hepatol. 2018

69. Abdel-Razik A, Mousa N, Shabana W, Refaey M, Elzehery R, Elhelaly R, et al. Rifaximin in nonalcoholic fatty liver disease: hit multiple targets with a single shot. Eur. J. Gastroenterol.Hepatol. 2018; 30:1237-46.

70. Wang W, Shi LP, Shi L, Xu L. Efficacy of probiotics on the treatment of non-alcoholic fatty liver disease. Zhonghua $\mathrm{Er} \quad \mathrm{Ke} \mathrm{Za}$ Zhi. 2018:57:101-6.

71. York LW, Puthalapattu S, Wu GY. Nonalcoholic fatty liver disease and low-carbohydrate diets. Annu. Rev. Nutr. 2009;29:365-79.

72. Adams LA, Angulo P. Treatment of non-alcoholic fatty liver disease Postgrad Med J. 2006;82:315-22.

73. Osaka T, Hashimoto Y, Hamaguchi M, Kojima T, Obora A, Fukui M Nonalcoholic fatty liver disease remission in men through regular exercise. J Clin Biochem Nu tr. 2018: 62:242-6.

74. Mazzella N, Ricciardi LM, Mazzotti A, Marchesini G. The role of medications for the management of patients with NAFLD. Clin Liver Dis. 2014;18:73-89.

75. Corrado RL, Torres DM, Harrison SA. Review of treatment options for nonalcoholic fatty liver disease. Med. Clin. 2014;98:55-72.

76. Fleet SE, Lefkowitch JH, Lavine JE. Current concepts in pediatric nonalcoholic fatty liver disease. Gastroenterol Clin. 2017;46:217-31.

77. Trappoliere M, Tuccillo C, Federico A, Di Leva A, Niosi M, D Alessio C,et al. The treatment of NAFLD. Eur. Rev. Med. Pharmacol. Sci. 2005;9:299

78. Hodson L, Bhatia L, Scorletti E, Smith DE, Jackson NC, Shojaee-Moradie F,et al. Docosahexaenoic acid enrichment in NAFLD is associated with improvements in hepatic metabolism and hepatic insulin sensitivity: a pilot study. Eur.J. Clin Nutr. 2017;71:973.

79. Orlando R, Azzalini L, Orando S, Lirussi F. Bile acids for non-alcoholic fatty liver disease and/or steatohepatitis. Cochrane Database Syst Rev. 2007.

80. Gabbi C, Carubbi F, Losi L, Loria P, Costantini M, Bertolotti M, Carulli N. Nonalcoholic fatty liver disease induced by leuprorelin acetate. J. Clin Gastroenterol. 2008;42:107-10.

81. Rafiq N, Younossi ZM. Effects of weight loss on nonalcoholic fatty liver disease. Semin. Liver Dis. 2008:28:427-433. 


\section{A Recent Research on Non Alcoholic Fatty Liver Disease}

82. Verrotti A, Di Marco G, La Torre R, Pelliccia P, Chiarelli F. Nonalcoholic fatty liver disease during valproate therapy. Eur.J Pediatr. 2009;168:1391-4.

83. El-Kader SM, Al-Shreef FM, Al-Jiffri OH. Biochemical parameters response to weight loss in patients with non-alcoholic steatohepatitis. Afr Health Sci.2016;16:242-9.

84. Sofer E, ShargorodskyM. Effect of metformin treatment on circulating osteoprotegerin in patients with nonalcoholic fatty liver disease. Hepatol Int. $2016 ; \mathbf{1 0 : 1 6 9 - 7 4}$

85. Ilan Y. Future of Treatment for Nonalcoholic Steatohepatitis: Can the Use of Safe, Evidence-Based, Clinically Proven Supplements Provide the Answer to the Unmet Need? Dig Dis Sci. 2018:11.

86. Paschos P, Paletas K. NAFLD and metabolic syndrome. Hippokratia. 2009;13:9.

87. Mikolasevic I, Milic S, Wensveen TT, Grgic I, Jakopcic I, Stimac D,et al Nonalcoholic fatty liver disease-A multisystem disease?.World J.Gastroenterol.2016;22:9488.

88. Munteanu MA, Nagy GA, Mircea PA. Current management of NAFLD. Clujul Med. 2016;89:19.

89. Hafeez S, Ahmed MH. Bariatric surgery as potential treatment for nonalcoholic fatty liver disease: a future treatment by choice or by chance?. J Obes. 2013: 29.

\section{AUTHOR PROFILE}



T. Panneerselvam received his $\mathrm{PhD}$ degree in 2013 from Acharya Nagarjuna University, Guntur, India and he earned Young Faculty Award 2014 from EET CRS presents Academic Brilliance Award-2014, Noida and has 10 years of teaching experience, scientific research and development. He is presently working as a Principal in Saraswathi College of Pharmacy.


A.Sankar Ganesh received B.Sc., in 1998 from Madras University and M Sc., in 2001 from Madras University. $\mathrm{He}$ is presently working in Kalasalingam Academy of Research and Education.
Cof Karavali College of Pharmacy who is pursuing internship in Father Muller Medical College Hospital Mangaluru. $\mathrm{He}$ has been doing research in the field of pharmacy and therapeutics since 2018

Deepak C G is a Pharm D student of Karavali College of Pharmacy who is pursuing internship in Father Muller Medical College Hospital Mangaluru. of pharmacy and therapeutics since 2018.

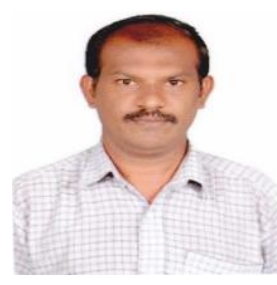

K. Selvaraj received B.Pharm. from Tamilnadu Dr. MGR Medical University in 1999, and Ph.D. from Jadavpur University., Kolkata in 2015. He is presently working as an Assistant Professor in Department of Biotechnology, Kalasalingam Academy of Research and Education, Tamilnad. He has won Erasmus Mundus Post- doctoral Fellowship, University of Warsaw, Poland. 